\title{
Inhaled Tranexamic Acid for Non-Massive Haemoptysis in a Rivaroxaban- Receiving Patient Not Responding to the Oral Form
}

\author{
Mugahid Eltahir ${ }^{1}$, Mohamed Nabil Elshafei $^{2}$, Abdelnasser Elzouki ${ }^{1,3}$ \\ ${ }^{1}$ Internal Medicine Department, Hamad General Hospital, Hamad Medical Corporation, Doha, Qatar \\ ${ }^{2}$ Clinical Pharmacy Department, Hamad General Hospital, Hamad Medical Corporation, Doha, Qatar \\ ${ }^{3}$ Weill Cornell Medicine-Qatar, Doha, Qatar
}

Doi: 10.12890/2020_001930 - European Journal of Case Reports in Internal Medicine - (c) EFIM 2020

Received: 01/08/2020

Accepted: 04/08/2020

Published: $30 / 12 / 2020$

How to cite this article: Eltahir M, Elshafei MN, Elzouki A. Inhaled tranexamic acid for non-massive haemoptysis in a rivaroxaban receiving patient not responding to the oral form. EJCRIM 2020;7: doi:10.12890/2020_001930.

Conflicts of Interests: The Authors declare that there are no competing interests.

This article is licensed under a Commons Attribution Non-Commercial 4.0 License

\section{ABSTRACT}

Haemoptysis is the expectoration of blood originating from the respiratory tract and occurs secondary to infection, malignancy, bronchiectasis or vascular disease. Its severity varies from minimal blood-streaked sputum to life-threatening haemorrhage. Therefore, prompt evaluation of the cause of the haemoptysis and its severity, and timely management are crucial. Although there is still no effective therapy for haemoptysis apart from treating the cause, inhaled tranexamic acid (TXA) might have a potential role in controlling non-massive haemoptysis. Here, we present a case of non-massive haemoptysis in a COPD patient with bronchiectasis on rivaroxaban for atrial fibrillation who responded to inhaled TXA following a failed trial of the oral formulation.

\section{LEARNING POINTS}

- Haemoptysis is the expectoration of blood and has several causes including bronchitis, bronchiectasis and bronchial neoplasms; severity ranges from simple blood-streaking of sputum to massive blood without sputum.

- Early recognition of haemoptysis, identification of the cause, and controlling it are crucial, especially in patients on anti-coagulation medicine.

- Inhaled tranexamic acid has shown benefit in controlling bleeding in patients with non- massive haemoptysis.

\section{KEYWORDS}

Tranexamic acid, haemoptysis, rivaroxaban, atrial fibrillation, COPD

\section{INTRODUCTION}

The term haemoptysis typically refers to the expectoration of blood originating from the lower respiratory tract. The most common causes of non-life-threatening haemoptysis in developed countries are acute bronchitis, bronchiectasis and bronchial neoplasms (primary or secondary) ${ }^{[1,2]}$. In contrast, in developing countries, infections due to Mycobacterium tuberculosis and Paragonimus westermani and non-cystic fibrosis-related bronchiectasis are more common causes ${ }^{[1,3,4]}$. Most episodes of haemoptysis are not life-threatening and originate from the pulmonary artery circulation, while life-threatening haemoptysis results in a life-threatening event including significant airway obstruction, significant abnormal gas exchange, or haemodynamic instability ${ }^{[5]}$. Initial management of haemoptysis is to ensure adequate oxygenation and ventilation, maintain the haemodynamic stability of the patient, and carry out initial investigations to identify the cause of bleeding and try to control it. 
Tranexamic acid (TXA) is an anti-fibrinolytic agent currently used systemically to control bleeding. It has also been described in case reports and a small randomized trial as reducing haemoptysis volume, and as resulting in faster resolution and a shorter hospital stay compared with treatment with inhaled normal saline ${ }^{[6,7]}$. In addition, a meta-analysis of two double-blind randomized controlled trials (RCTs) explored the effectiveness and safety of TXA administered via the intravenous or oral route compared with placebo in reducing the volume and duration of haemoptysis in adult and paediatric patients. The results demonstrated a significant reduction in bleeding time between patients receiving TXA and patients receiving placebo, with a weighted mean difference (WMD) of -19.47 ( $95 \% \mathrm{Cl}-26.90$ to -12.03 hours), but with high heterogeneity $\left(I^{2}=52 \%\right)$. TXA did not affect remission of haemoptysis evaluated at 7 days after the start of treatment. No adverse effects caused by the drug's mechanism of action were reported. There was no significant difference in the incidence of mild side effects between the active and placebo groups (OR 3.13, 95\% $\mathrm{Cl} 0.80$ to 12.24$)^{[8]}$.

Although there are promising data on the potential benefits of inhaled TXA, there is uncertainty regarding its benefit compared to intravenous or oral administration which mandates the need for larger RCTs.

\section{CASE DESCRIPTION}

We present the case of 63-year-old man with COPD/bronchiectasis and atrial fibrillation who was on rivaroxaban. The patient presented to our hospital with complains of shortness of breath, fever and desaturation. He was admitted as a case of acute exacerbation of COPD/ bronchiectasis, secondary to pseudomonas infection. He received anti-pseudomonal antibiotics and a 5-day course of steroids. His condition improved but he was dependent on oxygen and bilevel positive airway pressure (BiPAP) therapy as he had chronic CO2 retention with PCO2 of 94, and it was difficult for him to return home until a BIPAP device and oxygen could be provided for him there. On the 15th day of his stay, the patient developed mild haemoptysis which was attributed to be secondary to complications of bronchiectasis while he was on a direct oral anticoagulant (DOAC). Consequently, rivaroxaban was stopped for 1 day and the patient was closely observed. As his haemoptysis had not improved the next day, he was started on oral TXA $1 \mathrm{~g}$ every 8 hours which he continued to receive for 4 days during which time there was no significant improvement in his haemoptysis. The patient was then changed to inhaled nebulized TXA 500 mg every 8 hours. After 24 hours the patient noted improvement in his symptoms which completely resolved after 48 hours. He had a CT scan which showed mainly upper lobe cystic and varicoid bronchiectasis in both lungs (Fig. 1). He was restarted on a DOAC and did not report any other episodes of haemoptysis during 10 days of observation in hospital.

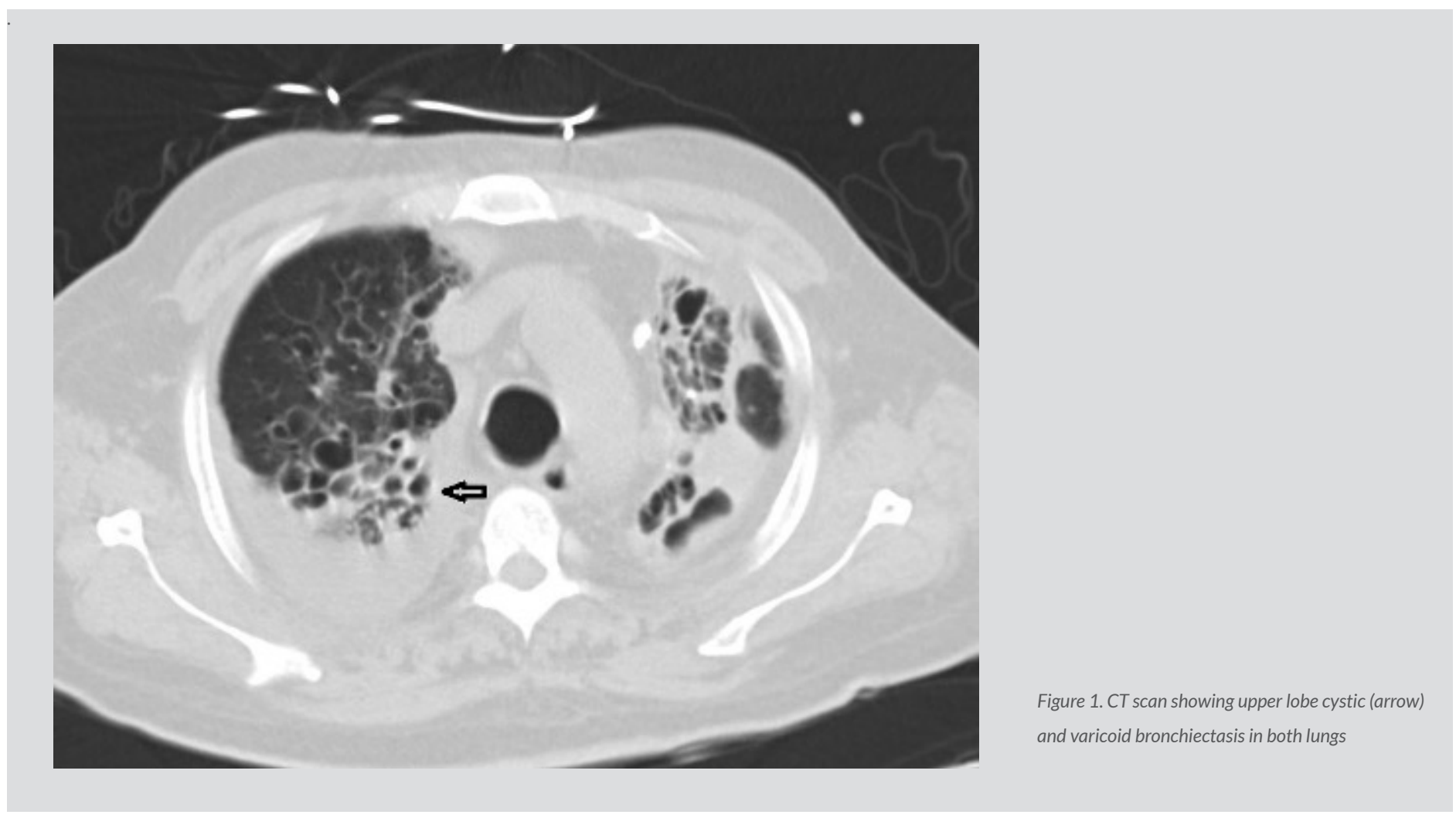




\section{DISCUSSION}

TXA has long been in use to control bleeding in various settings, primarily in its oral and intravenous forms. It has been well validated as an agent to control mucosal bleeding. The literature supporting the use of inhaled TXA as an agent to control haemoptysis is limited. Inhaled or endotracheal TXA seems to be effective and safe for pulmonary haemorrhage; recent RCT and case series have reported cessation of bleeding with inhaled TXA. Oral TXA has not been compared to inhaled TXA as both administration routes are thought to have similar effects. Inhaled aerosolized medications are routinely used for various chronic lung diseases such as COPD, asthma and bronchiectasis, and were shown to offer several advantages over systemically administered medication. These include more rapid onset of action for drugs such as $\beta$-adrenergic agonists compared with oral medication, high luminal doses for inhaled antibiotics when used to treat endobronchial infection, and an improved therapeutic index compared with systemic delivery for other classes of drugs such as corticosteroids ${ }^{[3,9,10]}$. Our patient showed complete resolution of haemoptysis after administration of nebulized TXA $5 \mathrm{mg}$ three times a day after failure to achieve significant improvement after 4 days of oral TXA. Although more evidence is needed to confirm the safety and effectiveness of TXA for the treatment of haemoptysis, our case has raised the question of whether there is any difference in the efficacy of inhaled compared to oral TXA.

\section{CONCLUSION}

Early recognition of haemoptysis, identification of the cause, and stopping the bleeding is essential, particularly in patients on anticoagulation medicine, who might have worse outcomes if haemoptysis remains uncontrolled. In patients with non-massive haemoptysis due to various causes, the use of inhaled TXA results in prompt resolution of haemoptysis compared to use of the oral form. However further studies are needed to confirm this finding.

\section{REFERENCES}

1. Johnston H, Reisz G. Changing spectrum of hemoptysis: underlying causes in 148 patients undergoing diagnostic flexible fiberoptic bronchoscopy. Arch Intern Med 1989;149(7):1666-1668.

2. Unsal E, Koksal D, Cimen F, Taci Hoca N, Sipit T. Analysis of patients with hemoptysis in a reference hospital for chest diseases. Tuberk Toraks 2006;54(1):34-42.

3. Santiago S, Tobias J, Williams AJ. A reappraisal of the causes of hemoptysis. Arch Intern Med 1991;151(12):2449-2451.

4. Prasad R, Garg R, Singhal S, Srivastava P. Lessons from patients with hemoptysis attending a chest clinic in India. Ann Thorac Med 2009;4(1):10.

5. Ibrahim WH. Massive haemoptysis: the definition should be revised. Eur Respir J 2008;32(4):1131-1132.

6. Wand O, Guber E, Guber A, Shochet GE, Israeli-Shani L, Shitrit D. Inhaled tranexamic acid for hemoptysis treatment: a randomized controlled trial. Chest 2018;154(6):13791384.

7. Dhanani JA, Roberts J, Reade MC. Nebulized tranexamic acid therapy for hemoptysis associated with submassive pulmonary embolism. J Aerosol Med Pulm Drug Deliv 2020;33(1):12-14.

8. Prutsky G, Domecq JP, Salazar CA, Accinelli R. Antifibrinolytic therapy to reduce haemoptysis from any cause. Cochrane Database Syst Rev $2012 ;(4)$ :CD008711.

9. Zhao Y, Li F, Liu Y, Shi Y, Li Z, Cao G, et al. Comparison of efficiency of inhaled and intravenous corticosteroid on pregnant women with COPD and the effects on the expression of PCT and hs-CRP. Exp Ther Med 2018;15(6):4717-4722.

10. Trend S, von Ungern, Sternberg BS, Devadason SG, Schultz A, Everard ML. Current options in aerosolised drug therapy for children receiving respiratory support. Anaesthesia 2017;72(11):1388-1397. 